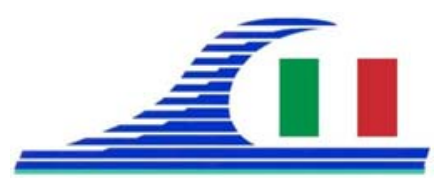

Conférence Méditerranéenne Côtière et Maritime EDITION 3, FERRARA, ITALIA (2015)

Coastal and Maritime Mediterranean Conference

Disponible en ligne - http://www.paralia.fr - Available online

\title{
Valorisation in situ des sédiments anthropiques des canaux du nord de la France
}

\author{
Philippe DHERVILLY ${ }^{1}$, Mickaël BERTRAND ${ }^{1}$, Daniel LEVACHER ${ }^{2}$
}

1. SEDIGATE, 17 rue Claude Bloch, BP 25102, 14000 Caen, France.

contact@sedigate.com; m.bertrand@sedigate.com

2. Université de Normandie, Unicaen, Laboratoire Morphodynamique Continentale et Côtière, UMR 6143 CNRS - M2C, 24 rue des Tilleuls, F-14000 Caen, France.

daniel.levacher@unicaen.fr

\section{Résumé :}

L'étude porte sur la valorisation des sédiments provenant d'un canal du nord de la France, précisément sur la rivière canalisée de la Lys. Pour atteindre cet objectif, plusieurs mélanges à base de liants pouzzolaniques ont été proposés et testés. L'ensemble des formulations ont fait l'objet d'une caractérisation mécanique simple par le biais d'essais de résistance en compression simple. Un minimum de résistance permet d'envisager des potentialités de valorisation matière. Ces mélanges ont été élaborés dans une démarche d'éco-conception pour aboutir à la stabilisation renforcée des sols de surface pour la réalisation de voies vertes, de vélo-routes ou de chemin de halage. Des éco-liants ont été développés à partir de la combustion de sous-produits industriels et permis la mise au point de divers procédés notamment les procédés ECOVOIE $^{\circledR}$ et ACTiV.SOL ${ }^{\circledR}$. Ces procédés ont été appliqués dans le cadre d'une valorisation matière des sédiments fluviaux comme matériaux routiers.

Mots-clés : Valorisation, Stabilisation, Solidification, Sédiment anthropique, Canaux, Liant.

\section{Introduction}

La société SEDIGATE explore depuis sa création les voies de co-valorisation possibles des matériaux fins en ingénierie routière. Dans cette étude, le matériau à recycler en question est un sédiment fluvial issu d'un cours d'eau du nord de la France, la Lys. Elle prend sa source à Lisbourg dans les collines de l'Artois et fait partie du bassin versant Artois-Picardie. La Lys, avec $85 \mathrm{~km}$ parcourus dans sa partie française, est divisée en deux tronçons: la Lys rivière et la Lys canalisée (GABELLE, 2006). C'est dans ce dernier tronçon qu'ont été prélevés les sédiments en divers points. L’étude porte sur un échantillon moyen pour représenter la totalité des sédiments de ce tronçon.

La valorisation matière de ces sédiments fins repose sur l'application d'une technique de stabilisation et/ou solidification. Ainsi plusieurs formulations sont expérimentées avec un liant à prise pouzzolanique, élaboré à base de coproduits industriels, i.e. des cendres volantes et autres additifs. Les performances mécaniques de ces mélanges sont 
Côtes méditerranéennes menacées :

Risques et défis dans le contexte du changement climatique

évaluées à partir d'essais de résistance en compression simple. Elles permettent d'étudier le comportement de ces sédiments stabilisés et/ou solidifiés en vue d'une réutilisation en technique routière comme matériau alternatif. Ils pourront ainsi remplacer certaines couches constituant la structure d'une chaussée, comme la couche de base ou mieux, la couche de roulement.

\section{Méthodologie}

\subsection{Caractéristiques physico-chimiques}

Toute valorisation nécessite la détermination des caractéristiques physico-chimiques du matériau i.e. le sédiment fluvial. Elles sont reportées dans le tableau 1. Les différents échantillons prélevés dans le canal ont été homogénéisés pour ne faire qu'un seul sédiment à traiter comme dans la pratique. En effet, cette étape permet de simuler le mélange des différents sédiments stockés dans un bassin, lors des différentes campagnes de dragage effectuées sur le même canal. L’homogénéisation des sédiments a été faite sur site dans des proportions égales par le gestionnaire du canal, puis réceptionnés comme sédiment moyen.

Tableau 1. Propriétés physico-chimiques du sédiment moyen du canal de La Lys.

\begin{tabular}{lll}
\hline Paramètres & Méthodes & Matériau moyen \\
\hline Matière organique (\%) & Perte au feu à $550^{\circ} \mathrm{C}$ & 9 \\
\hline & Tamisage et laser & \\
\multirow{2}{*}{ Nature granulométrique (\%) } & Argiles : $0<d<2 \mu \mathrm{m}$ & 2 \\
& Limons : $2<d<63 \mu \mathrm{m}$ & 62 \\
& Sables : $63 \mu \mathrm{m}<d<2 \mathrm{~mm}$ & 36 \\
\hline
\end{tabular}

La caractérisation montre que la teneur en matière organique de $9 \%$ du sédiment moyen est élevée. Généralement pour un sol ou sédiment, nous pouvons considérer qu'une teneur en matière organique est élevée à partir du moment où celle-ci est supérieure à 5\% (TROALEN et al., 1996). L'ajout d'un additif a été nécessaire pour neutraliser la matière organique présente en quantité non négligeable. L’analyse granulométrique révèle un matériau limono-sableux, les particules de dimensions comprises entre 2 et 63 $\mu \mathrm{m}$ représentent $62 \%$ des constituants et celles comprises entre $63 \mu \mathrm{m}$ et $2 \mathrm{~mm}, 36 \%$. Ces sables peuvent contribuer à la résistance.

\subsection{Mise en œuvre des formulations}

L'étude d'éco-conception d'un matériau alternatif menée sur ces sédiments de canal tend à limiter le dosage en liants pouzzolaniques : le dosage reste compris entre 8 et $12 \%$. De récentes études menées en laboratoire et sur sites ont déjà abouti à de bonnes performances quant à l'utilisation de liants pouzzolaniques pour une valorisation de sédiments marins en technique routière (BEHMANESH, 2008 ; SILITONGA et al., 
2010 ; MIRAOUI, 2010 ; OBANA et al., 2010). Pour la stabilisation/solidification du sédiment moyen du canal de la Lys, des mélanges ternaires - sédiment-liant-additif ont été étudiés selon les formulations du tableau 2. La confection d'éprouvettes cylindriques, de $34 \mathrm{~mm}$ de diamètre et d'un élancement de 2 est nécessaire pour réaliser les essais de résistance. La teneur en eau $\mathrm{W}$ des sédiments incorporés dans les mélanges était de $30 \%$, soit une siccité de $76 \%$. Nous rappelons la relation entre la teneur en eau W (\%) et la siccité S (\%) qui s’écrit comme suit :

$W=\frac{W_{e}}{W_{s}}$ et $S=\frac{W_{e}}{W_{t}}$

avec We, Ws, Wt comme étant les masses respectives d'eau, de matière sèche et totale.

La condition de cure retenue pour les échantillons est une cure à l'air qui correspond à la mise en place de matériaux de surface dans le domaine voirie routière. Les échantillons ont donc été conservés sur paillasse à l'air libre à une température voisine de $20^{\circ} \mathrm{C}$. Les essais de résistance en compression simple ont été réalisés sur ces échantillons et ce, pour l'ensemble des mélanges étudiés. Ils ont été effectués à des dates conventionnelles de 7, 14 et 28 jours. Les teneurs en liants et additif données dans le tableau 2, sont exprimées en pourcentage de la masse du sédiment sec. Le liant pouzzolanique est une cendre volante silico-alumineuse capable de stabiliser des sables et des sédiments fins de manière durable. L'additif utilisé est un hydroxyde de calcium, i.e. de la chaux. Cette chaux est utilisée pour réduire l'interférence de la matière organique sur les réactions pouzzolaniques des cendres volantes, d'une part. Elle permet d'autre part d'activer ces réactions. Le choix des formulations $F_{i}$ a été fait en fonction d'une addition croissante de liant pouzzolanique jusqu'à $12 \%$ (tableau 2) et l'incorporation de l'additif à un taux constant de 1.5\%. L'échantillon témoin $T$ ne concerne aucun traitement du sédiment. L’indicateur mécanique déterminant évalué est la résistance mécanique à la compression simple $R c$ dont le seuil retenu est de $1 \mathrm{MPa}$ à 28 jours de cure (GTR SETRA-LCPC, 2000). Ce seuil a permis de valider les formulations en comparant les valeurs de $R c$ et le développement de ces performances à la compression.

Tableau 2. Formulations proposées.

\begin{tabular}{lll}
\hline Références & Liant pouzzolanique (\%) - LP & Additif (\%) - A \\
\hline Témoin T & 0 & 0 \\
Formulation F1 & 8 & 1.5 \\
Formulation F2 & 10 & 1.5 \\
Formulation F3 & 12 & 1.5 \\
\hline
\end{tabular}

\section{Résultats}

Les résultats des essais de compression simple sur les mélanges ont permis d'établir les constats suivants, voir figure 1. L'évolution dans le temps des résistances $R c$ des 
Côtes méditerranéennes menacées :

Risques et défis dans le contexte du changement climatique

formulations F1, F2, et F3 présente une tendance classique avec des valeurs croissantes durant 28 jours de cure. Le mélange témoin $T$ qui correspond au sédiment non traité montre toute l'efficacité des différentes formulations étudiées. Les résistances moyennes à la rupture à 28 jours, $R_{c 28}$ atteignent respectivement 2.5, 2.8 et 2.7 MPa. Les formulations ne montrent pas de palier de résistance à 28 jours. Si les formulations Fi permettent d'atteindre une valeur de $1 \mathrm{MPa}$ dès 14 jours, elles sont relativement proches à 28 jours. Même à 7 jours, elles sont, juste en deçà de la valeur seuil de $1 \mathrm{MPa}$. La formulation $\mathrm{F} 1$, quel que soit l'âge, fournit des $R c$ inférieures aux deux autres formulations. Pour ces dernières, on semble tendre vers un optimum de résistance pour un dosage en liant pouzzolanique croissant.

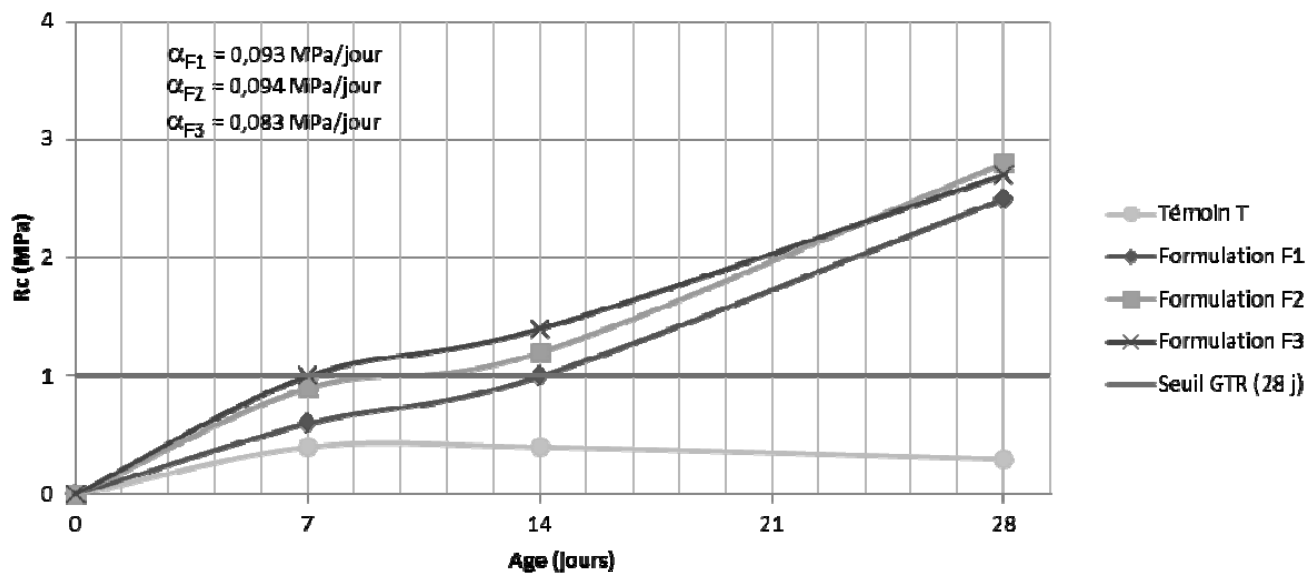

Nota : $\alpha_{F i}$ sont les pentes des droites ajustées pour chaque formulation

Figure 1. Evolution des résistances en compression simple.

Comme observé sur la figure 1, l'ensemble des formulations fournissent des résistances suffisantes, i.e. $\geq 1 \mathrm{MPa}$, en compression simple dès 14 jours de cure. Rappelons que ce seuil de résistance fixé à $1 \mathrm{MPa}$ à 28 jours de cure correspond à un à un seuil de traficabilité qui permet d'éventuelles circulations d'engins de chantier. Finalement, la formulation retenue pour une application en technique routière est la formulation F2 qui respecte les caractéristiques souhaitées, avec une incorporation moyenne de liant dans le sédiment, à savoir $10 \%$ de $\mathrm{LP}+1.5 \%$ de chaux.

La figure 2 montre l'évolution des différentes valeurs de $R c$ obtenues selon les formulations F1, F2 et F3 à 28 jours de cure. Les données confirment la tendance observée précédemment, i.e. une évolution croissante de $R c$ avec la quantité de liants pouzzolaniques ajoutée dans les mélanges. Mais une tendance asymptotique amène à dire qu'un pourcentage maximal de liants est atteint vers $10 \%$. Cela peut signifier que le liant n’a pas pu complètement être hydraté et/ou qu'il a été utilisé en excès dans le mélange. Cela est observé par une amorce de décroissance de la résistance à 28 jours du mélange (figure 1). 
On peut aussi à partir d'un ajustement polynomial, en considérant la valeur de la formulation témoin $\mathrm{T}$ du sédiment non traité, approcher plus précisément le taux minimal de liant pouzzolanique à ajouter pour obtenir au moins 1 MPa de résistance en compression (BERTRAND et al., 2014).

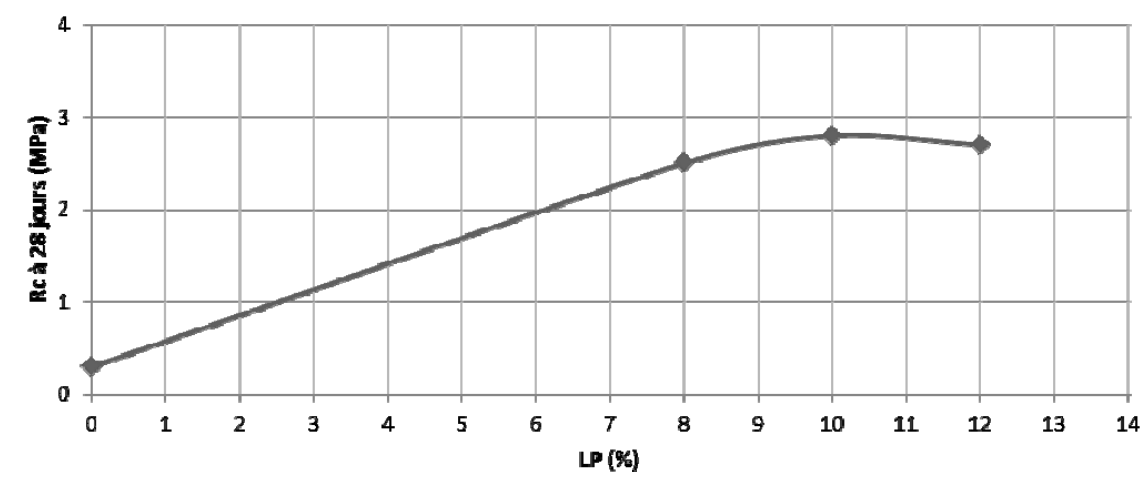

Figure 2. Evolution des valeurs de Rc à 28 jours en fonction du dosage en liants pouzzolaniques (LP).

\section{Conclusions et perspectives}

Différents sédiments ont été prélevés, puis rassemblés pour être homogénéisés afin d'obtenir un seul et même sédiment dit moyen, représentatif du tronçon-canal de la Lys. C'est à partir de ce sédiment moyen qu'une étude de stabilisation et solidification a été menée. La formulation F2 qui incorpore une quantité raisonnable i.e. 10\% de liant pouzzolanique dans le sédiment, sera préférée à la formulation $F 1$, car elle possède dès 7 jours des performances satisfaisantes. Ceci permet dans le cas d'un chantier en technique routière une réouverture rapide de la voie. Les autres atouts de ces formulations sont les critères techniques, économiques et écologiques que possèdent les liants pouzzolaniques présents dans les mélanges. Les formulations Fi sont à base de cendres volantes. Une cendre volante dégage moins de $\mathrm{CO}_{2}$ qu'un constituant usuellement utilisé dans des formulations de stabilisation pour les sédiments qu'est le ciment. Ce constat est fait par l'observation du bilan carbone réalisé pour la fabrication des deux liants comparés. En effet, un ciment (type CEM II) est fabriqué à base de clinker (65 à 94\%), de cendres volantes (6 à 35\%) et d'autres constituants secondaires $(\leq 5 \%)$. Le bilan carbone pour la fabrication du ciment est plus élevé que les cendres volantes, puisqu'elles font partie de la composition du ciment (CEM II). Les cendres volantes ont un bilan carbone 3 à 4 fois moins élevé que le ciment pour des incorporations dans les mélanges équivalents. La formulation retenue F2 est donc plus écologique qu'une formulation classique à base de ciment. Cette formulation F2 covalorise un coproduit industriel, un liant pouzzolanique; ceci correspond bien à la notion de gestion durable. Un traitement de sédiments stockés sur un même site peut 
Côtes méditerranéennes menacées :

Risques et défis dans le contexte du changement climatique

être envisagé pour une valorisation en technique et voirie routière. Ainsi, les différents sédiments extraits du tronçon étudié de la Lys pourront être réutilisés comme couche de base en chemin de halage par exemple. Ceci réduit les coûts élevés de transport et de production de matière première à $8 \%$ environ. Ce traitement peut se faire en masse, procédé usuellement appliqué par la société SEDIGATE à des sédiments avec des liants pouzzolaniques. Deux procédés sont proposés utilisant des liants pouzzolaniques référencés : ACTiV.SOL ${ }^{\circledR}$ traitement en centrale ou ECOVOIE ${ }^{\circledR}$ traitement sur site. C’est ce dernier procédé qui peut être envisagé dans le cas présent. Ce même procédé fera l'objet d'applications sur une plateforme expérimentale COVASED de regroupement et de traitement de sédiments, (DHERVILLY et al., 2014).

\section{Références bibliographiques}

BEHMANESH J. (2008). Etude de la durabilité d'un sédiment, traité au ciment et additifs. Thèse de doctorat, Université de Caen, 215 p.

BERTRAND M., DHERVILLY P., LEVACHER D. (2014). Valorisation des fines de dragage en Manche. XIII ${ }^{\text {èmes }}$ Journées Nationales de Génie Côtier - Génie Civil, Dunkerque, pp 969-976. http://dx.doi.org/10.5150/jngcgc.2014.107

DHERVILLY P., BERTRAND M., THANNEBERGER L., LEVACHER D., HOUISE C., LAFHAJ Z. (2014). Gestion durable des sédiments : le projet COVASED. XIII ${ }^{\text {èmes }}$ Journées Nationales de Génie Côtier - Génie Civil, Dunkerque, pp 977-986. http://dx.doi.org/10.5150/jngcgc.2014.108

GABELLE C. (2006). Etude de la contamination des sédiments par les métaux dans les canaux et rivières du Nord de la France. Thèse de doctorat, Université de Lille, 22 p.

GTR SETRA-LCPC. (2000). Traitement des sols à la chaux et/ou aux liants hydrauliques, Application à la réalisation des remblais et des couches de forme, $108 \mathrm{p}$.

MIRAOUI M. (2010). Mise en ouvre d'une démarche de prétraitement et de traitement des sédiments de dragage en vue d'une valorisation dans le génie civil. Thèse de doctorat, Université de Lille, 210 p.

OBANA M., LEVACHER D., DHERVILLY P. (2010). Durability properties of marine sediments stabilized by pozzolan and alkali activated binders. XI ${ }^{\text {èmes }}$ Journées Nationales de Génie Côtier - Génie Civil, Sables d’Olonne, pp 887-894. http://dx.doi.org/10.5150/jngcgc.2010.096-O

SILITONGA E., LEVACHER D., MEZAZIGH S. (2010). Utilization of fly ash for stabilization of marine dredged sediments. European Journal of Environmental and Civil Engineering, Vol. 14, n 2, pp 253-265. http://dx.doi.org/10.3166/ejece.14.253-265

TROALEN J.P., DURON C., HAGNERE C. (1996). Traitement de vases de l'estuaire de la Seine, $4^{\text {èmes }}$ Journées Nationales Génie Civil Génie Côtier, Dinard, France, pp 499-506. http://dx.doi.org/10.5150/jngcgc.1996.048-T 